\title{
The Seeds of Dissolution: Discrepancy and Incoherence in Buyer-Supplier Exchange
}

\author{
Qiong Wang \\ Smeal College of Business, Pennsylvania State University, University Park, Pennsylvania 16802, qiongwang@psu.edu \\ Ujwal Kayande \\ Research School of Business, Australian National University, Acton, Australian Capital Territory 0200, Australia, \\ ujwal.kayande@anu.edu.au \\ Sandy Jap \\ Goizueta Business School, Emory University, Atlanta, Georgia 30322, sjap@emory.edu
}

\begin{abstract}
Tn this research, we examine a novel mechanism of interorganizational relationship dissolution: incoherence in Ia partner's behavior. We propose that the discrepancy between an exchange partner's opportunistic behavior and the focal firm's expectations may create a state of incoherence and uncertainty and that this effect can be damaging to the exchange even when the partner's behavior is better than expected. Using nearly 500 longitudinal, confidential reports of industrial buyers and sellers, we find supportive evidence that (1) the net effect of the discrepancy is initially positive when behavior is better than expected but becomes rapidly negative thereafter, and (2) the net effect of the discrepancy is always negative when behavior is worse than expected. Thus, these effects will generally damage the exchange even as the partner tries to improve the relationship. This gives insight into why exchange relationships that hit a downward spiral can be difficult, if not impossible, to salvage. We also show that the dysfunctional consequences of discrepancy are mitigated through exchange structures such as the magnitude of dependence on an organizational partner, the development phase of the relationship, and the presence of bilateral idiosyncratic investments. Implications for theory and the management of interorganizational relationships are developed.
\end{abstract}

Key words: interorganizational relationship management and performance; belief discrepancy; incoherence; organizational sensemaking; opportunism; dependence; relationship phase; bilateral investments

History: Received: August 25, 2008; accepted: April 5, 2010; processed by Jinhong Xie. Published online in Articles in Advance June 24, 2010.

\section{Introduction}

Exchange partners do not always behave as expected, and this could be good, bad, or neutral for the exchange. One would expect that the impact of such behavioral discrepancies would correspond-i.e., relationships should suffer when expectations are not met, remain neutral when met, and improve when exceeded. Instead, we surprisingly find that when the partner behaves too desirably, i.e., as the discrepancy increases in a direction better than expected, the relationship actually suffers. Using nearly 500 confidential, longitudinal reports from industrial buyers and sellers, we show that the impact of positive discrepancies from expectations, although initially positive, becomes rapidly negative thereafter. In other words, it is as if a partner's extraordinarily good behavior is penalized.

We propose a novel mechanism for this effect, suggesting that the discrepancy between a focal firm's belief-based expectations and actual behavior facilitates a state of incoherence and uncertainty, whose impact on interorganizational exchange performance goes beyond the simple linear effect of the discrepancy. Incoherence requires that new information be assimilated with extant beliefs and expectations and sets in motion a phenomenon commonly known as "sensemaking," or a search for meaning (Thomas et al. 1993). Hence, we propose (1) a more nuanced specification of expectation that stems from the focal firm's general beliefs about the market and specific beliefs about its exchange partner, and (2) that a better specification of the impact of discrepancy should involve the simple linear effect, as well as a quadratic effect, for the accompanying incoherence that is generated. One implication of this specification is that the resulting uncertainty from any discrepancy, positive or negative, could undermine the firm's evaluations of exchange performance and its intentions to remain in the exchange, and this is in fact what we show.

We couch our investigation in the context of ex post opportunism, a topic of growing interest in the interorganizational management literature in marketing. See 
Wathne and Heide (2000) for a comprehensive review of this literature and subsequent empirical studies (Brown et al. 2000; Jap and Anderson 2003, 2007). Specifically, we propose that a firm forms an expectation of opportunism based on (i) its belief about the relationship between opportunism and goal congruence in exchange relationships and (ii) its perception of congruence of goals in the exchange. We find that opportunism that deviates from such belief-based expectations (the deviation is hereafter called belief discrepancy) has a negative quadratic impact on performance above and beyond its linear impact, regardless of whether opportunism is less (or more) than expected and despite the best (or worst) efforts of the partner firm. This result is consistent with our argument that discrepancy creates uncertainty and also suggests that incoherence, like gravity, facilitates exchange along a downward spiral that is difficult to counter. We highlight three boundary conditions that can mitigate the impact of these negative effects on exchange performance, including (i) the focal firm's dependence on the partner firm, (ii) the early stages of relationship development, and (iii) mutual commitments to idiosyncratic investments.

We are the first to examine discrepancy and incoherence in an interorganizational context and make several contributions. We introduce and explore the role of organizational beliefs and their constraining impact on exchange performance. Thus, we go beyond examining the impact of behavior or each party's perceptions of the other, which has been the standard approach. By bridging research on customer preference formation with organizational sensemaking, we highlight a fundamental mechanism contributing to relationship dissolution. Moreover, our focus on the beliefs and inferences of a single party in a relationship is consistent with the viewpoint that relationship dissolution is fundamentally different from buildup (Duck 1981), as it requires only one participant rather than both parties.

In the next section, we introduce the notion of discrepancy and formulate hypotheses for its nonlinear impact on performance evaluations and continuity intentions. We also identify boundary conditions for this effect. An empirical test of the hypotheses on industrial buyers and sellers in ongoing relationships is described. This paper concludes with a discussion of results, limitations, and implications for management and future research.

\section{Conceptualization}

Our unit of analysis is a focal firm's (either a buyer or a seller) view of an ongoing business relationship. In this section, we develop the notion of discrepancy and incoherence, and we examine its impact on the exchange. We then follow with a discussion of potential boundary conditions.

\section{Incoherence and Belief Discrepancy}

Central to our paper is the notion that organizations have beliefs about how interorganizational relationships work, and any discrepancy from belief-based expectations creates incoherence that generates uncertainty. Incoherence has recently been examined in the product management literature (Kayande et al. 2007) and has been studied extensively in the behavioral sciences (e.g., Murphy and Medin 1985, MeyersLevy and Tybout 1989, Rehder and Hastie 2004). At its core is the assumption that, through previous knowledge and experience, consumers form beliefs about interattribute covariation in a product category. Such beliefs, right or wrong, form through empirical observation or subjective theories and are difficult to change (Broniarczyk and Alba 1994). A second critical assumption is that consumers use these beliefs as the basis for inferences (cf. Dick et al. 1990). Thus, incoherence arises when the consumer faces attribute information (e.g., a product claim) far from what they would expect, given their interattribute covariation beliefs and the level of the other attributes. The focus of these studies is on how incoherent information is assimilated and its net effect on preference.

We propose that organizations respond similarly to discrepant information and engage in a phenomenon widely known in the organizational literature as sensemaking (Thomas et al. 1993). This theory suggests that the firm regularly scans the environment for information, then interprets and acts to improve performance (Daft and Weick 1984). An observed discrepancy with beliefs triggers sensemaking: "Explicit efforts at sensemaking tend to occur when the current state of the world is perceived to be different from the expected state of the world, or when there is no obvious way to engage the world" (Weick et al. 2005, p. 409).

We examine these processes and investigate their potential effects in the context of interorganizational opportunism. A central premise of transaction cost economics is that opportunism is the chief risk in transactions; economic actors will act opportunistically if given the chance to do so (Williamson 1993). ${ }^{1}$ Opportunism is self-interest seeking with guile, and it creates maladapted, unsustainable transactions; it limits both parties' potential gains and can lead to redistribution of value. We view the firm's beliefs about the nature of opportunism and its covariates as the genesis of the firm's expectations from its partner.

Also figuring prominently in discussions of opportunism is goal congruence, or the degree to which both parties perceive the possibility of achieving compatible, if not identical, objectives. Transaction cost

\footnotetext{
${ }^{1}$ We acknowledge that there are alternative candidates for explaining relationship dissolution; however, we focus on opportunism as a first step to illustrating the effects of incoherence and encourage future research to explore alternative possibilities.
} 
economists and agency theorists view goal congruence as central to curbing opportunism (Eisenhardt 1989). Goals are generally stable over time and can account for much variance in organizational outcomes (Nilakant and Rao 1994). Firms may pursue both economic goals (such as maximizing profits or utility) and noneconomic goals (such as social goals-the development of trust and commitment, sociability, approval, status, and power), and the partners in exchange may pursue quite different goals (Eliashberg and Michie 1984). We focus not on the nature of each party's goals per se but the extent to which both party's goals can be mutually accomplished in the exchange. Thus, we regard the focal firm's general belief structure regarding the relationship between goal congruence and opportunism as fundamental to the future direction, nature, and scope of actions in the exchange both initially and over time.

Consistent with the literature, we propose that the focal firm $i$ forms a belief, based on market experience, of how goal congruence inhibits opportunism. We represent $i$ 's belief of the relationship between opportunism (OPPT) and goal congruence (GOAL) as follows:

$$
O P P T_{i j}=\beta_{0 i}+\beta_{1 i} \cdot G O A L_{i j}+\varepsilon_{i j},
$$

where $O P P T_{i j}$ is $i$ 's perception of partner $j$ 's opportunism, $G O A L_{i j}$ is $i$ 's perception of the congruence of goals between $i$ and $j, \beta_{0 i}$ represents information that $i$ has about any partner's average opportunistic behavior, $\beta_{1 i}$ is a partial regression coefficient (representing $i$ 's belief about how opportunism is inhibited by goal congruence), and $\varepsilon_{i j}$ indicates that goal congruence and opportunism may not be perfectly correlated. Consistent with Kayande et al. (2007), we assume that $\varepsilon_{i j}$ is normally distributed with mean 0 and that $\beta_{0 i}$ and $\beta_{1 i} \cdot G O A L_{i j}$ are uncorrelated.

Firm $i$ forms an expectation of a specific partner $j$ 's opportunistic behavior at time $t$, denoted by $E\left[O P P T_{i j, t}\right]$, based on the belief structure specified in Equation (1) and its current perception of the congruence of the two parties' goals $\left(G O A L_{i j, t}\right)$ at time $t$ as follows:

$$
E\left[O P P T_{i j, t}\right]=\beta_{0 i}+\beta_{1 i} \cdot G O A L_{i j, t} .
$$

Thus, we specify expectations about the behavior of a specific partner as a function of two components: (1) $i$ 's belief of how goal congruence is generally related to opportunism in a relationship (i.e., $\beta_{0 i}$ and $\beta_{1 i}$ ) and (2) $i^{\prime}$ s perception of how congruent $j^{\prime} s$ goals are with its own (i.e., $G O A L_{i j, t}$ ). The first component is market specific, whereas the second component is partner specific. This nuanced explanation of expectation formation represents a departure from the predominant view of expectations in marketing-i.e., a linear disconfirmation model in which experiences that fall short of expectations reduce satisfaction (a disappointment effect) and experiences that exceed expectations improve satisfaction (a positive surprise or delight effect). This literature views expectations as a function of the consumer's experience and personal needs, as well as word-of-mouth communication (Parasuraman et al. 1985). In contrast, we suggest that expectations are the result of a belief process. We will subsequently show that such a viewpoint provides a better fit with data than using expectations based on past experience alone. We refer to the departure in observed opportunism from a belief-based expectation as a discrepancy (DISCREP):

$$
D_{S C R E P} P_{i j, t}=O P P T_{i j, t}-E\left[O P P T_{i j, t}\right] .
$$

We also differ from the norm in the satisfaction literature by modeling the impact of discrepancy with a linear and quadratic effect. ${ }^{2}$ Discrepant information (positive or negative) can generate uncertainty and ambiguity (Einhorn and Hogarth 1985); the "unexpectedness" of observing a deviation from beliefbased expectations increases uncertainty because it conflicts with held beliefs about interattribute covariation. Kayande et al. (2007) were the first to propose the quadratic specification and demonstrate its negative effects on risk-adjusted preferences. Geylani et al. (2008), in a study of co-branding, derived a similar quadratic effect of the difference in attribute performance of the two constituent brands on uncertainty about a focal brand's attribute performance. This uncertainty's effect must be accommodated in preference formation of risk-averse entities (Chiles and McMackin 1996). Thus, the weighted sum of the linear and quadratic effects on performance represents the net effect of discrepancy, as follows:

$$
\begin{aligned}
& \text { NET DISCREP } P_{i j, t} \\
& \quad=\tau_{0}+\tau_{1} \text { DISCREP }_{i j, t}+\tau_{2} \text { DISCREP }_{i j, t}^{2} .
\end{aligned}
$$

We expect the linear effect to be positive and, consistent with our uncertainty argument, the quadratic effect to be negative. The latter is because Keeney and Raiffa (1993) have shown that risk-averse entities discount uncertainty. Most economic and statistical theories of decision making assume that decision makers are risk averse over the entire range of possible outcomes (Arrow 1971) and that this perspective is carried through to organizations.

\section{Performance Outcomes}

We consider two key performance outcomes: the focal firm's overall evaluation of its partner's performance and the focal firm's continuity intentions, ${ }^{3}$ both of

\footnotetext{
${ }^{2}$ The satisfaction literature has also proposed a quadratic effect; however, their conceptualization of its role is that of saturation (Mittal et al. 1998, Kopalle and Lehman 2001).
} 
which have received considerable attention in the interorganizational literature. The firm's overall evaluation of partner performance reveals "a shadow of the past," whereas the focal firm's continuity intentions reflect "a shadow of the future" (Poppo et al. 2008, p. 39).

Overall Evaluation of Partner Performance. Evaluation of partner performance is a summary assessment of the partner's value in the relationship to date (Kumar et al. 1992). It reflects the view of the focal firm in the form of an individual, global assessment of the current balance of benefits, and costs of working with a specific partner. In short, a positive evaluation of partner performance is needed to justify the focal firm's involvement in the business relationship.

Continuity Intentions. The focal firm's continuity intentions are a critical harbinger of anticipated actions and volitions. Bercovitz et al. (2006) found in a research and development (R\&D) context that a party's willingness to collaborate in the future was a key predictor of actual future collaboration, which suggests that intentions are the basis for the plans of commitment in the future. Such intentions have also been linked to lower negotiation costs (Artz and Brush 2000), reduced opportunism (Jap and Anderson 2003), and stronger relational ties between organizations (Joskow 1987). Continuity intentions form a foundation, the basis of a focal firm's willingness to engage in processes and investments for the long run (Anderson and Weitz 1989, Heide and Miner 1992).

Given our conceptualization of discrepancy and the incoherence that arises from this unexpected observation, we expect that performance outcomes will be undermined. This is because firms (and decision makers within those firms) are risk averse, and uncertainty increases the potential risk associated with exchange. The literature on organizational theory also suggests that uncertainty, or the belief that a situation "feels different," is experienced similarly and will likely lead to a discount being applied (Weick et al. 2005 , p. 414). Hence, we expect the following.

Hypothesis 1 (H1). Belief discrepancy has a negative quadratic effect on the focal firm's evaluations of partner's performance and continuity intentions.

\section{Boundary Conditions on Incoherence}

Past research has shown that the most important predictors of interorganizational relationship dissolution are the structure, processes, and actions of the

\footnotetext{
${ }^{3}$ We would have preferred to observe actual continuity, but this is typically impossible in organizational research, and secondary data sets at the relevant unit of analysis-a focal firm's vieware typically unavailable. This construct is analogous to the notion of "expectations of continuity," but we drop the use of the term "expectations" here to avoid confusion with the expectations embedded in our measure of belief discrepancy.
}

exchange parties, whereas the competitive environment plays a considerably weaker role (Baker et al. 1998). Thus, we focus on three boundary conditions figuring prominently in the literature on interorganizational management: (i) the focal firm's dependence on its partner, (ii) the extent of the relationship's development, and (iii) bilateral, idiosyncratic investments in the exchange. Together, they reflect the relationship structure of the exchange, its level of development, and the economic stakes of the partners, respectively. All three are strategic levers for the design and crafting of successful interorganizational exchange relations and have been widely studied.

Although these factors can be viewed as various forms of switching costs, the conceptual mechanism behind this differs for each. For example, the net effect of the focal firm's dependence on its partner is that it remains in the exchange because it has to-there are no better alternatives. In contrast, developing a relationship over time motivates both firms to remain because the learning and experience curves can be further exploited. In contrast, bilateral investments create significant economic risks for both parties. Because belief discrepancy is a novel construct to the interorganizational literature, we consider all three mechanisms to validate how discrepancy might be related (but distinct) to the existing nomological network.

Own Dependence on Partner. Power and its flip side, dependence, are central constructs in channel management. Dependence refers to the degree to which a firm relies on its counterpart for the performance of joint functions, as well as access to scarce resources (see Lusch and Brown 1996). Thus, the focal firm must maintain the relationship to achieve its desired goals. As the magnitude of dependence increases, the more dependent firm lacks control over the exchange and is more likely to tolerate the uncertainty stemming from its high-power partner's behavior. A potential counterargument is that highpower partners can be unpredictable, which increases exchange uncertainty, undermining relationship outcomes. On balance, however, we expect the following.

Hypothesis $2(\mathrm{H} 2)$. The negative quadratic effect of belief discrepancy on evaluations of partner performance and continuity intentions is mitigated (becomes less negative) as the focal firm's dependence on the partner increases.

Relationship Development. The interorganizational exchange literature has had a long-standing interest in how relationships develop over time, beginning with the seminal work of Dwyer et al. (1987), who outlined the processes, behaviors, and outcomes that develop systematically across stages of the relationship: awareness, exploration, buildup, maturity, decline, and deterioration. As relationships develop, there is a base of actual experience that upholds or updates the focal firm's beliefs. Hence, belief 
structures tend to crystallize and become stronger. We propose that the negative effect of discrepancy may loom larger in more-developed relationships as deviations in opportunism from expectations create a relatively starker contrast to the focal firm's entrenched belief structure.

Hypothesis 3 (H3). The negative quadratic effect of belief discrepancy on evaluations of partner performance and continuity intentions worsens (becomes more negative) in more-developed (i.e., mature) exchanges.

Bilateral Investments. Bilateral investments are nonfungible economic resources (e.g., dedicated personnel, capital equipment, machinery, or processes). Mutual investments on both sides of the dyad represent credible, mutual commitments with economic stakes (Anderson and Weitz 1992). By "binding the hands of both parties," the partners are incentivized to act in their mutual best interests over their individual interests. Hence, bilateral investments create strong incentives for relationship continuity and cooperation, thus mitigating the negative effect of discrepancy on performance outcomes.

Hyротнеsis 4 (H4). The negative quadratic effect of belief discrepancy on evaluations of partner performance and continuity intentions is mitigated (becomes less negative) in the presence of bilateral investments in the exchange.

Collectively, these hypotheses suggest that the net effect of these moderators is to shift the point at which uncertainty weighs in on the exchange. The focal firm's dependence on its partner or joint investments from both sides of the dyad cause the focal firm to tolerate more belief discrepancy, whereas developed relationships will tolerate less. Thus, the moderators change the impact of the quadratic term, whereas the linear effect of discrepancy remains constant.

\section{Methodology}

\section{Data Collection and Sample}

The hypotheses were tested via a longitudinal survey of interorganizational relationships across four Fortune 50 manufacturing firms: a computer (PC) manufacturer, a photography equipment manufacturer, a chemical manufacturer, and a brewery. Participation was solicited in exchange for customized analyses, presentation of results, and an executive summary brief. All measures were collected in two waves with the same key informants, who report on the view of their firm. We used data on goal congruence and opportunism from the first wave to calibrate the focal firm's belief structure (as per Equation (1)) and then used estimates of $\beta_{0 i}$ and $\beta_{1 i}$ to construct a measure of discrepancy in the tests of hypotheses on data collected a year later.

To leverage the data opportunity we had with these four Fortune 50 firms, we asked the participant firms to request participation from both the buyer and supplier in each exchange to maximize the potential sample size while minimizing potential attrition in the second wave of data collection. Hence, across the four firms, 200 buyers were asked to report on two supply relationships, creating a dyadic sampling frame of 400 relationships. This approach allows us to increase our sample size power, but it creates potential dependence between observations from the same dyad, which we will address in our model estimation. We also test for whether there are any systematic differences across buyers and sellers in the impact of discrepancy.

Procedure. Buyers were mailed questionnaires, a preaddressed, postage-paid envelope, a cover letter from the researchers, and a memorandum from corporate executives requesting participation and assuring the confidentiality of their individual responses. Buyers were instructed to identify a supply relationship and a contact individual at the supplier firm as a reference point for completing the questionnaire. Upon receipt of the buyer surveys, a parallel survey identifying the buyer firm and the individual respondent was sent to the counterparts in supplier firms. Hence, both the buyer and supplier used the other (their counterpart) as a reference point. In the second data collection wave, questionnaires were mailed to all the buyers and suppliers identified in the first wave. A nonresponse bias test rejects the null hypothesis that there is a response difference for early versus later respondents (Armstrong and Overton 1977).

Sample Characteristics. Two hundred seventy-five buyer surveys were returned at time 1 (a 69\% response rate; note that the 200 buyers in our sampling frame were asked to report on their relationships with two suppliers). This allowed the mailing of 275 corresponding supplier surveys, of which 220 were completed (an $80 \%$ response rate). Buyers who responded in time 1 were contacted once again a year later, with 167 total surveys returned (a 61\% response). Similarly, suppliers who responded in time 1 were also surveyed a year later, resulting in 154 completed supplier surveys (a 70\% response). Our sample from time 1 consisted of 492 observations, with 350 respondents. The final sample in time 2 had 321 observations, with 239 respondents. Buyers and suppliers had worked with each other an average of 9.1 years. Annual transactions involved over $\$ 99$ million in materials and services, such as capital equipment, components, services, maintenance supplies, repair supplies, and operating supplies. Collectively, these sample characteristics suggest that the 
relationships have a significant history base and a variety of exchange experience.

Respondent Competence. Our hypotheses rely on individual respondents' perceptions of the focal firm's relationship with a partner. Hence, we examined both global and specific measures of their ability to serve as key informants. The global measures included the respondents' professional experience in their field of work and within their firm. Buyer respondents averaged 11.2 years of experience in their area and had been with their companies 20.9 years on average. Supplier respondents averaged 15.1 years of experience and 14.2 years of employment with their companies. Multiple-item measures assessed the respondent's specific knowledge of the focal relationship. ${ }^{4}$ Responses were indicated on a 7-point scale $(1=$ not very knowledgeable, $7=$ very knowledgeable). The sample mean for this scale was 5.40, with a standard deviation of 1.16 and a range of 1 to 7 . Together, these global and specific measures provided some assurance that the key informants were competent, knowledgeable, and relatively involved in completing the survey.

Common Method Bias and Demand Effects. Because the independent and dependent measures for the model were collected from the same respondent, the potential for demand effects is created. Although this was mitigated to some degree by the use of a longitudinal design, it was also impossible that one respondent (let alone over 200) might guess our proposed specification of discrepancy and its moderating effects. However, we did take a number of steps to reduce the potential for common method bias according to the prescriptions of Podsakoff et al. (2003) and Rindfleisch et al. (2008), such as verifying that our key informants were knowledgeable, guaranteeing respondent anonymity by mixing specific measurement items so that they were not adjacent to each other, and asking respondents to report on observable aspects (e.g., specific investments, relationship length, opportunistic behavior, etc.) of the relationship (Crampton and Wagner 1994). ${ }^{5}$

We also conducted statistical tests to assess whether a single common factor better accounts for

\footnotetext{
${ }^{4}$ This was assessed via a variety of items such as "How knowledgeable are you about the following in your firm's relationship with the buyer/supplier firm?" Below were listed items such as "how similar their goals are," "the nature of unique investments, assets, capabilities, etc. that are used in the relationship," or "the degree to which they have earned strategic advantages over their competitors."

${ }^{5}$ We also assessed page-by-page analyses of potential order effects and insensitivity to reverse score items. Each page demonstrated significant variation from item to item, suggesting that on average, respondents did not take a lackadaisical view of their item completion and simply mark the same response down the page in a straight line.
}

the data than our more complex model (Lindell and Whitney 2001, Podsakoff et al. 2003). This first-order, latent factor model was estimated via full-information maximum-likelihood techniques in LISREL 8.8 (Jöreskog and Sörbom 2006). The $\chi^{2}$ of $5,805.38$ (527 df) rejects the null hypothesis that a single factor better fits the data, and the overall fit indices drop from an average of 0.96 for our proposed (more) complex model structure to 0.82 for the one factor model (both the comparative fit index (CFI) and incremental fit index (IFI) are 0.82, whereas the TuckerLewis index (TLI) is 0.81). The parsimony-adjusted root mean square error of approximation (RMSEA) for the single factor model is 0.21 , nearly three times that of our proposed model structure (a satisfactory fit is typically reflected around 0.08 , with a very good fit reflected at 0.05 ). The individual loadings on a single factor are much lower (sometimes as low as 0.31 versus the typical 0.6 rule-of-thumb standard) than in the more complex model structure. Collectively, these results provide confidence that common method bias was likely minimized.

\section{Questionnaire and Scale Development}

Relationship phase (RELPHASE) was measured using the measures developed by Jap and Ganesan (2000), which consisted of a dummy variable coded as 1 if the relationship was in the mature, decline, or deterioration phases to indicate relationships that were more developed (0 otherwise). Fifty-four percent of the sample was coded as being in more-developed relationships.

Scale Items. Constructs were measured via multiple-item, 7-point scale measures reflecting the view of the focal firm. Although each scale contains seven response points, the measure of each construct reflects the mean of multiple items, resulting in a large number of response points, or a close to continuous scale. ${ }^{6}$ The scale items and reliabilities are presented in the appendix. The estimated reliabilities for the constructs were high, averaging 0.81 , with a range of 0.66 to 0.91 . Construct means, standard deviations, and correlations are presented in Table 1. Together, there is significant heterogeneity in the range of values reflected by these constructs.

Measurement Model. A covariance matrix of the observable indicators was used to estimate a latent factor measurement model comprised of all constructs. Factor loadings, measurement errors, and correlations of and between each construct were in acceptable ranges and significant at $\alpha=0.05$, providing evidence of convergent validity. The overall chi-square for this model is 1,798.72 (499 df, $p<0$ ). The CFI and the IFI are 0.96; the TLI is 0.95. The RMSEA is 0.075,

\footnotetext{
${ }^{6}$ We also tested the models using an ordinal specification, and the results are substantively unchanged.
} 
Table 1

Descriptive Statistics of Key Constructs $(N=321)$

Correlation between variables ${ }^{\mathrm{a}}$

\begin{tabular}{|c|c|c|c|c|c|c|c|c|c|c|c|c|}
\hline Variable & Min & $\operatorname{Max}$ & Mean & Std. dev. & 1 & 2 & 3 & 4 & 5 & 6 & 7 & 8 \\
\hline 1. PERFEVAL & 1.33 & 7.00 & 5.38 & 1.20 & & & & & & & & \\
\hline 2. CONTIN & 1.00 & 7.00 & 5.84 & 1.12 & 0.71 & & & & & & & \\
\hline 3. GOAL & 2.00 & 7.00 & 5.12 & 0.94 & 0.71 & 0.61 & & & & & & \\
\hline 4. NEGDISCREP & -3.08 & 0.00 & -0.33 & 0.54 & 0.32 & 0.22 & 0.17 & & & & & \\
\hline 5. POSDISCREP & 0.00 & 2.02 & 0.32 & 0.43 & -0.02 & -0.01 & -0.10 & 0.46 & & & & \\
\hline 6. DEPEND & -3.95 & 2.93 & 0.00 & 1.13 & 0.03 & -0.01 & 0.01 & -0.03 & -0.05 & & & \\
\hline 7. INVEST & 1.75 & 7.00 & 5.32 & 1.11 & 0.56 & 0.55 & 0.48 & 0.06 & -0.27 & 0.02 & & \\
\hline 8. RELLNGTH & 0.00 & 67.00 & 9.11 & 10.2 & 0.00 & 0.02 & -0.03 & 0.07 & 0.09 & 0.02 & -0.10 & \\
\hline 9. $P R O B F R E Q$ & 1.00 & 7.00 & 2.84 & 1.30 & -0.41 & -0.31 & -0.37 & -0.32 & -0.17 & 0.09 & -0.10 & -0.08 \\
\hline
\end{tabular}

Note. We do not present correlations for relationship development phase, which is a dummy variable (mean $=0.54$ ).

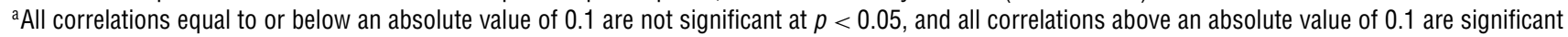
at $p<0.05$.

suggesting a satisfactory fit of the model in relation to its degrees of freedom. Discriminant validity was assessed (and met) between every possible pair of constructs via the procedure recommended by Fornell and Larcker (1981).

Measure of Own-Dependence Construct. Earlier, we argued that the focal firm's dependence on the partner mitigates the negative quadratic effect of discrepancy on performance due to the firm's lack of influence and power over the partner. Conceptually then, our construct should capture this structural dependence of the focal firm on its partner, rather than the mutual dependence of the two partners on each other. The latter might be influenced by bilateral idiosyncratic investments. Empirically, we find that the measures of dependence and bilateral idiosyncratic investments are correlated at 0.41 , which makes estimation problematic. Thus, we parse out the mutual dependence in our dependence magnitude measure by first regressing this measure on bilateral idiosyncratic investments. ${ }^{7}$ The predicted values of dependence capture the mutual dependence of both parties in the relationship as a result of the bilateral idiosyncratic investments. The residual component from this regression can then be construed as a measure of the focal firm's own dependence, free of any mutual aspects (DEPEND). A useful by-product of this procedure is also the elimination of collinearity between the two measures and between their respective interactions with the negative quadratic effect of belief discrepancy.

Face Validity. Our empirical analysis focuses on the role and impact of belief discrepancy. In our conceptualization, we assume on the basis of findings reported in the literature that some variables are

\footnotetext{
${ }^{7}$ In this regression, we follow the model estimation approach described later in this paper for estimating the models in Equations (5) and (9), both of which account for potential correlations between observations in our data. The intercept and slope in this regression were 1.99 and 0.44 , respectively, and both were significant at $p<0.01$.
}

related. To this end, we verified these assumptions via a series of individual regressions among these variables. $^{8}$ We found that goal congruence does curb opportunism $(\beta=-0.56, p<0.001)$, and opportunism negatively influences the focal firm's evaluation of partner performance (PERFEVAL) $(\beta=-0.14$, $p<0.001)$ and continuity intentions (CONTIN) $(\beta=-0.12, p<0.001)$, as expected. The focal firm's continuity intentions are positively related to evaluations of partner's performance $(\beta=0.71, p<0.05)$. In regard to switching costs, bilateral investments (INVEST) facilitate continuity intentions $(\beta=0.71$, $p<0.001)$ but are not significantly related to evaluations of partner performance $(\beta=-0.36$, n.s.).

\section{Analysis}

We conducted a two-stage analysis to test our hypotheses. In the first stage, we estimated the focal respondents' marketplace beliefs of how opportunism is related to goal congruence, based on their past experiences in multiple relationships. In the second stage, we used those beliefs along with perceptions of goal congruence with a partner to construct current expectations of that partner's opportunistic behaviors and measures of discrepancy and its quadratic effect, and to test their net effect on performance outcomes.

Stage 1: Calibrating Belief Structures. Our belief structure formation model in Equation (1) is an individual-level model. Some buyers reported on multiple suppliers, and all those suppliers reported on the said buyer. Thus, there is significant potential for dependence between observations from such "connected" buyers and suppliers. Additional dependence is potentially introduced by the buyers being located in one of four manufacturing firms.

To account for this potential observational dependence, we first define a network to be a buyer and

\footnotetext{
${ }^{8}$ We were unable to enter these effects directly into our empirical examination of incoherence, as these factors also comprise the discrepancy measures and the correlations between them make the overall equation inestimable.
} 
all their suppliers. We assume all respondents within a network have the same belief structure. ${ }^{9}$ This is reasonable, because a network consists of a unique buyer who is reporting on one or more suppliers, and those suppliers reporting on their relationship with the same focal respondent (i.e., the buyer). There are 136 such networks in our data. We specify the following mixed-effects model (Searle et al. 1992) for the focal respondent $i$ 's perception of the opportunism displayed by partner $j$, with respondent $i$ and partner $j$ nested within the $k$ th network ( $k=1$ to 136$)$ and the $l$ th manufacturing firm $(l=1$ to 4$):{ }^{10}$

$$
O P P T_{i j: k l}=\beta_{0}+\beta_{1} \cdot G O A L_{i j: k l}+\varepsilon_{i j: k l},
$$

where

Assumption I. $\beta_{0}=\bar{\beta}_{0}+u_{0 k}$ (where $\bar{\beta}_{0}$ is the average intercept across all respondents, $u_{0 k}$ is the random networkspecific intercept, and $u_{0 k} \sim N\left(0, \sigma_{u_{0}}^{2}\right), k=1$ to 136).

Assumption II. $\beta_{1}=\bar{\beta}_{1}+u_{1 k}$ (where $\bar{\beta}_{1}$ is the average slope across all respondents, $u_{1 k}$ is the random networkspecific slope, and $u_{1 k} \sim N\left(0, \sigma_{u_{1}}^{2}\right), k=1$ to 136).

Assumption III. $\varepsilon_{i j: k l} \sim N(0, \Sigma)$.

Assumption IV. $\sum$ is the block diagonal error covariance matrix of all observations, with diagonal elements $\sum_{l}$, which is the error covariance matrix of observations located in the lth firm $(l=1$ to 4$)$.

Assumption V. $\sum_{l}$ is a positive definite matrix with $\left(\sigma_{e}+\sigma_{l}\right)$ as diagonal elements, $\sigma_{l}$ as off-diagonal elements, and $\sigma_{e}>\sigma_{l}$.

Assumptions (IV) and (V) specify a nondiagonal error covariance structure for the fixed effects and account for potential dependence in observations that come from buyers (and associated suppliers) from within each of the four firms. We assume that there is a firm-specific common correlation across all observations within a firm, observations across firms are uncorrelated, and that the random effects and error term are uncorrelated.

Equation (5) allows us to estimate unique belief structures at the network level, assuming thereby that all individual respondents within a network have identical belief structures. For any individual

\footnotetext{
${ }^{9}$ We evaluated this assumption by calculating the average withinnetwork variance and average between-network variance for the belief structure variables opportunism and goal congruence. The ratio of between-network variance to total variance (i.e., the sum of between- and within-network variance) was $75 \%$ and $78 \%$ for opportunism and goal congruence, respectively, suggesting that respondents within a network have similar perceptions of opportunism and goal congruence and that those perceptions are different, on average, from those in other networks.

${ }^{10}$ In contrast to the theoretical model in Equations (1) and (2), we are now modeling the individual respondents as key informants of the focal firm.
}

respondent $i$ in the $k$ th network, the intercept and slope of their belief structure are

$$
b_{0 i: k l}=\bar{\beta}_{0}+u_{0 k}, \quad \text { and } \quad b_{1 i: k l}=\bar{\beta}_{1}+u_{1 k} .
$$

In all subsequent discussions, we simplify the subscript $i: k l$, referring to it as only $i$. Our model approach allows us to estimate 136 belief structures, which were substantially heterogeneous in both intercept (ranging from 3.15 to 5.95, with a mean of 4.52) and slope (ranging from -0.69 to -0.28 , with a mean of -0.48 ). We then used these estimated belief structures to construct our measure of belief discrepancy, discussed next, for tests of hypotheses.

Stage 2: Testing the Hypotheses. We now use the belief structures estimated in Equations (5) and (6) to first construct an expectation of opportunism at current time $t$ as per Equation (2):

$$
E\left[O P P T_{i j: k l, t}\right]=b_{0 i}+b_{1 i} \cdot G O A L_{i j: k l, t} .
$$

Our measure of discrepancy is

$$
\operatorname{DISCREP} P_{i j: k l, t}=O P P T_{i j: k l, t}-E\left[O P P T_{i j: k l, t}\right] .
$$

Similar to Hardie et al. (1993) conceptualization of asymmetric effects in the loss and gain domains, we allowed for the effect of discrepancy to vary by defining the following two new variables, negative belief discrepancy (NEGDISCREP) and positive belief discrepancy (POSDISCREP):

$$
\begin{gathered}
\text { NEGDISCREP } P_{i j: k l, t}=O P P T_{i j: k l, t}-E\left[O P P T_{i j: k l, t}\right] \\
\text { if } O P P T_{i j: k l, t}>E\left[O P P T_{i j: k l, t}\right], E L S E=0 ; \\
\text { POSDISCREP }_{i j: k l, t}=O P P T_{i j: k l, t}-E\left[O P P T_{i j: k l, t}\right] \\
\text { if } O P P T_{i j: k l, t} \leq E\left[O P P T_{i j: k l, t}\right], E L S E=0 .
\end{gathered}
$$

Because opportunism is a negatively valenced variable, we reverse code the above two variables in our analysis to aid interpretation of the coefficients. Tests of hypotheses are conducted using the following model specification for firm $i$ 's evaluation of partner $j$ 's performance at time $t$ (for simplicity, we drop the subscript $i j: k l, t$, which applies to all the variables in the following equation):

$$
\begin{aligned}
& \text { PERFEVAL } \\
& =\gamma_{0}+\gamma_{1} \cdot G O A L+\gamma_{2} \cdot N E G D I S C R E P \\
& +\gamma_{3} \cdot \text { POSDISCREP }+\gamma_{4} \cdot(\text { NEGDISCREP })^{2} \\
& +\gamma_{5} \cdot(\text { POSDISCREP })^{2}+\gamma_{6} \cdot \text { DEPEND } \\
& +\gamma_{7} \cdot D E P E N D \times(D I S C R E P)^{2} \\
& +\gamma_{8} \cdot \text { RELPHASE }+\gamma_{9} \cdot \text { RELPHASE } \times(\text { DISCREP })^{2} \\
& +\gamma_{10} \cdot I N V E S T+\gamma_{11} \cdot \operatorname{INVEST} \times(\text { DISCREP })^{2} \\
& +\gamma_{12} \cdot \text { RELLNGTH }+\gamma_{12} \cdot \text { PROBFREQ. }
\end{aligned}
$$


We do not expect the effect of moderators to vary in the negative and positive discrepancy domains, so we did not include asymmetry in those effects (strong empirical support for this assumption is reported later in this paper). We estimated the model in Equation (9) with the focal firm's continuity intentions as a second dependent variable. In estimating these two models, we included a network-specific random intercept term to account for dependence in observations within a network and incorporated the same nondiagonal error covariance structure as in Equation (5) to account for dependence in observations within each of the four firms. ${ }^{11}$ We also included two covariates, the frequency of problems in the exchange relationship (PROBFREQ) and the length of the relationship (RELLNGTH) in years. Although this latter construct may appear conceptually similar to the development phase, empirically they can vary significantly. Like the product life cycle, a development phase may or may not coincide with the actual length of the relationship. Additionally, the prevailing view in the interorganizational management literature is that relationship length and phase are different concepts but can co-exist. ${ }^{12}$

\section{Results}

A summary of the results are presented in Table 2. We note that goal congruence perceptions are significantly related to the focal firm's evaluation of partner's performance $(0.59, p<0.01)$ and continuity intentions (0.39, $p<0.01)$, consistent with the notion of goal congruence as a key safeguarding mechanism of performance outcomes. In the domain of positive discrepancy (opportunism less than expected), an increase in discrepant behavior leads to a positive linear effect (0.71 and 0.76, respectively, for evaluation of partner performance and continuity intentions; $p<0.05$ ), as expected. However, in the same domain, an increase in discrepant behavior leads to a negative quadratic effect $(-0.66$ and -0.68 , respectively, for evaluation of partner performance and continuity intentions; $p<0.01$ ), supporting H1. The size of the quadratic effect is almost as much as the size of the linear effect, indicating that the negative quadratic will rapidly overcome the linear positive effect in influencing performance outcomes. This finding is counterintuitive, as conventional wisdom would suggest that being less

\footnotetext{
${ }^{11}$ We incorporate this model structure to account for dependence among observations in all analyses from here on, including robustness checks. As an additional check, we also estimated a model with a diagonal error covariance structure and firm-specific dummies as fixed effects. The results are substantively the same as reported in Table 2.

${ }^{12}$ As a check, we regressed relationship phase on relationship length (via a binary logit model) and found that they were not related in our data.
}

opportunistic is better for exchange performance. Our results suggest that, in fact, such actions can have very detrimental effects. In the domain of negative discrepancy, we find that as the negative discrepancy worsens, evaluation of partner performance deteriorates linearly $(0.48, p<0.05)$ but without an observable negative quadratic effect. In contrast, continuity intentions deteriorate in a quadratic fashion $(-0.46, p<$ 0.05 ), supporting $\mathrm{H} 1$, but without an observable linear effect. Note that the net effect in the negative discrepancy domain was predicted to be negative throughout, which is what we observe. However, in the negative discrepancy domain, it is probably more difficult to disentangle the linear and quadratic effects as they are both negative. ${ }^{13}$ In the positive domain, the two terms have contrasting effects-i.e., the linear is positive and the quadratic is negative.

Boundary Conditions. Hypotheses $\mathrm{H} 2$ to $\mathrm{H} 4$ were tested by estimating the relationships between the quadratic effect of discrepancy and performance outcomes among the three moderating conditions. Own dependence significantly moderates (reduces) the quadratic effects on continuity intentions $(0.12$, $p<0.01)$ whereas the same for evaluation of the partner's performance is marginal $(0.07, p<0.10)$. This suggests increasing tolerance of the uncertainty associated with discrepancy as the focal firm's dependence on its partner increases. Hence, $\mathrm{H} 2$ is supported.

Similarly, the stage of relationship development exacerbates the dysfunctional effects of belief discrepancy; the more mature the business relationship, the more negative the impact of the quadratic on evaluation of partner's performance $(-0.18, p<0.05)$ and continuity intentions $(-0.27, p<0.01)$. Thus, $\mathrm{H} 3$ is supported.

Last, bilateral idiosyncratic investments have a positive effect on the evaluation of partner's performance (0.27, $p<0.01)$ and continuity intentions $(0.32, p<$ $0.01)$, consistent with the past literature that underscores the role of these investments in enhancing exchange outcomes (e.g., Anderson and Weitz 1992, Jap 1999, Jap and Anderson 2003). Moreover, the negative quadratic effect of discrepancy on performance outcomes is mitigated by the presence of these investments. Specifically, the greater the investment, the less severe the negative quadratic effect of discrepancy on the evaluation of partner's performance $(0.05$, $p<0.10)$ and continuity intentions $(0.08, p<0.05)$. Therefore, H4 is supported.

\section{Robustness Checks}

As with most modeling and empirical analyses, we made assumptions and choices related to the measurement of our constructs, model, analysis, and our

\footnotetext{
${ }^{13}$ If the range of our data in the negative discrepancy domain were greater, then we might be able to observe these separate effects.
} 
Table 2 Effect of Incoherence on Performance Outcomes

\begin{tabular}{|c|c|c|c|}
\hline Variable & $\begin{array}{l}\text { Evaluation of } \\
\text { partner's performance }\end{array}$ & $\begin{array}{l}\text { Continuity } \\
\text { intentions }\end{array}$ & Result \\
\hline GOAL & $0.59 * \quad(10.82)$ & $0.39 * \quad(7.03)$ & \\
\hline NEGDISCREP & $0.48^{* *}(2.04)$ & $-0.20 \quad(-0.82)$ & \\
\hline POSDISCREP & $0.71^{* *}(2.04)$ & $0.76^{\mathrm{a}, *}(2.11)$ & \\
\hline \multicolumn{4}{|l|}{$\mathrm{H} 1$} \\
\hline $\begin{array}{l}(\text { NEGDISCREP })^{2} \\
(P O S D I S C R E P)^{2}\end{array}$ & $\begin{array}{ll}-0.10 & (-0.4) \\
-0.66^{\mathrm{a}, *} & (-2.59)\end{array}$ & $\begin{array}{ll}-0.46^{* *} & (-1.86) \\
-0.68^{*} & (-2.72)\end{array}$ & Supported \\
\hline DEPEND & $0.01 \quad(0.12)$ & $-0.03 \quad(-0.75)$ & \\
\hline \multicolumn{4}{|l|}{$\mathrm{H} 2$} \\
\hline DEPEND $\times(D I S C R E P)^{2}$ & $0.07^{* * *}(1.6)$ & $0.12^{*} \quad(2.43)$ & Supported \\
\hline RELPHASE & $0.06 \quad(0.61)$ & $0.03 \quad(0.33)$ & \\
\hline \multicolumn{4}{|l|}{$\mathrm{H} 3$} \\
\hline RELPHASE $\times(\text { DISCREP })^{2}$ & $-0.18^{* *}(-2.02)$ & $-0.27^{*}(-2.94)$ & Supported \\
\hline INVEST & $0.27^{*} \quad(5.19)$ & $0.32^{*} \quad(6.08)$ & \\
\hline \multicolumn{4}{|l|}{$\mathrm{H} 4$} \\
\hline$I N V E S T \times(D I S C R E P)^{2}$ & $0.05^{* * *}(1.35)$ & $0.08^{* *}(2.06)$ & Supported \\
\hline RELLNGTH & $0.00 \quad(0.52)$ & $0.00 \quad(1.18)$ & \\
\hline PROBFREQ & $-0.13^{*} \quad(-3.68)$ & $-0.10^{*} \quad(-2.6)$ & \\
\hline Intercept & $2.22^{*} \quad(5.71)$ & $1.32^{*}(3.47)$ & \\
\hline AIC & 762 & 790 & \\
\hline
\end{tabular}

Note. All coefficients are unstandardized; $t$-statistics in parentheses.

a Indicates a significant difference between coefficients in the positive and negative domains.

${ }^{*} p<0.01,{ }^{* *} p<0.05,{ }^{* * *} p<0.10$, all one-tailed tests.

sample. Although our model specification has a good theoretical pedigree, we verified that (i) the belief structures underlying our measure of discrepancy are relatively stable over time, (ii) our quadratic specification of discrepancy provides an improved explanation of performance outcomes, (iii) our assumption of the source of expectations is better than alternative assumptions, (iv) the effect of discrepancy is similarly manifested across the dyad, and (v) our measure of discrepancy is correlated yet distinct enough from related measures such as trust.

First, our theory suggests belief structures are a critical component of expectations of partner behavior. An implicit assumption is that these belief structures are relatively stable over time, in particular because of their more general nature. To test whether this is the case, we applied the same mixed-effects estimation to obtain each respondent's belief structure coefficients at time 2 (as specified in Equations (5) and (6)). We can compare the underlying belief structures of respondents who responded across both time periods to examine the stability of their belief structure. Because the two-dimensional nature of the belief structure (i.e., an intercept and slope) precludes a simple comparison, we estimated measures of discrepancy based on belief structures calibrated with time 1 and time 2 data. The correlation in measures of discrepancy across two time periods is $0.86\left(R^{2}=0.74\right)$, indicating that our measure is relatively stable over time.

Second, we defined the impact of discrepancy as both a linear and quadratic effect, but it remains to be seen whether this specification improves model fit. Hence, we considered three alternative models: (1) a linear-only model (i.e., with a discrepancy term but no quadratic), (2) a model without a discrepancy effect (either linear or quadratic), and (3) a model that includes interactions with a linear effect (in place of interactions with the quadratic). Table 3 gives an overview of the results. Our proposed model fits the data better than all three alternative models. Akaike's information criterion (AIC) for all three of these alternative models ranges from 770 to 777 (versus 762 for our proposed model) for evaluation of the partner's performance and from 797 to 808 for continuity intentions (versus 790 for our proposed model).

These results collectively provide support for the quadratic effect of discrepancy from expectations. We note that our model includes asymmetric effects for positive and negative discrepancy with respect to the linear and quadratic effects. However, we also find that it outperforms a fully asymmetric model-i.e., asymmetries represented in every term involving discrepancy-including the moderator effects (AIC $=780$ and 812 for evaluation of partner performance and continuity intentions, respectively) as well as a fully symmetric model (AIC $=767$ and 792 for evaluation of partner performance for continuity intentions, respectively).

Third, we proposed that the focal firm's expectations are a function of both its perceptions of goal congruence with a specific partner and its belief of 


\begin{tabular}{|c|c|c|c|}
\hline \multirow[b]{2}{*}{ Test explanation } & \multirow[b]{2}{*}{ Alternative models } & \multicolumn{2}{|c|}{ Performance outcome ${ }^{a}$} \\
\hline & & $\begin{array}{l}\text { Evaluation of } \\
\text { partner's performance }\end{array}$ & $\begin{array}{l}\text { Continuity } \\
\text { intentions }\end{array}$ \\
\hline & $\begin{array}{l}\text { Our proposed quadratic model—containing the linear and } \\
\text { quadratic effects of discrepancy }\end{array}$ & 762 & 790 \\
\hline Is our quadratic model better than a linear-only model? & $\begin{array}{l}\text { A linear-only model with a discrepancy term, but no } \\
\text { quadratic term }\end{array}$ & 770 & 808 \\
\hline $\begin{array}{l}\text { Is our model better than one without any deviation } \\
\text { effect? }\end{array}$ & A model without a linear or quadratic term & 777 & 799 \\
\hline $\begin{array}{l}\text { Is our model better than one with interactions with the } \\
\text { linear effect? }\end{array}$ & $\begin{array}{l}\text { A model in which the interactions with the quadratic are } \\
\text { replaced with interactions with the linear effect }\end{array}$ & 771 & 797 \\
\hline
\end{tabular}

how goal congruence is generally related to opportunism in exchange relationships. We now consider how our specification compares to alternatives, such as whether expectations might be better represented as only (1) past behavior (i.e., the perspective of the satisfaction literature), (2) absent the quadratic term, or (3) as random. Table 4 provides an overview of the results.

In every case, our proposed model outperforms the possible alternatives; the AIC for evaluation of partner's performance in these alternative models ranges from 790 to 828 (versus 762 in our proposed model) and from 805 to 846 for continuity intentions (versus 790 in our proposed model), providing confidence in our results. Furthermore, in the model in which expectations are defined as past behavior, we find a positive linear effect of discrepancy, but we do not find a quadratic effect in our data. Such a result substantively implies that behavior better than expected is rewarded by the focal firm without any limits. In contrast, we model how beliefs constrain the actions of partner firms by inducing an uncertainty penalty when partner firms deviate from belief-based expectations. Our model outperforms the naïve model on fit with the data, indicating that discrepant behavior is penalized severely even if it is better than expected.

More generally, we tested our proposed model against a range of 27 other models that varied in their characterization of asymmetry across negative and positive discrepancy (fully asymmetric, fully symmetric, or asymmetric only in linear and quadratic terms), functional form (linear only, or linear and quadratic), expectations (belief based, past experience based, or random), and whether an interaction of moderators with the linear effect was included (yes or no). This set of models represents a relevant set of models that we could test our model against, and we found that our proposed model provides the best fit with the data.

Fourth, we examined the possibility that the quadratic impact of discrepancy might be differentially represented across the dyad-i.e., that buyers,

Table 4 Assessment of Alternative Specifications of Expectations

\begin{tabular}{|c|c|c|c|}
\hline \multirow[b]{2}{*}{ Validation of specification of expectations } & \multirow[b]{2}{*}{ Alternative models } & \multicolumn{2}{|c|}{ Performance outcome $^{a}$} \\
\hline & & $\begin{array}{l}\text { Evaluation of } \\
\text { partner's performance }\end{array}$ & $\begin{array}{l}\text { Continuity } \\
\text { intentions }\end{array}$ \\
\hline & $\begin{array}{l}\text { Our proposed quadratic model—containing the linear } \\
\text { and quadratic effects of discrepancy }\end{array}$ & 762 & 790 \\
\hline $\begin{array}{l}\text { Is our model better than one with the expectation } \\
\text { defined as the partner's past opportunistic } \\
\text { behavior? }\end{array}$ & $\begin{array}{l}\text { Model with linear and quadratic effects of discrepancy, } \\
\text { where discrepancy is defined as } \\
{\left[O P P T_{i j: k l, t}-O P P T_{i j: k l, t-1}\right]}\end{array}$ & 794 & 810 \\
\hline $\begin{array}{l}\text { Is our model better than one with the expectation } \\
\text { defined as the partner's past opportunistic } \\
\text { behavior, and without the quadratic term? }\end{array}$ & $\begin{array}{l}\text { Model with linear effect of discrepancy, where } \\
\text { discrepancy is defined as }\left[O P P T_{i j: k l, t}-O P P T_{i j: k l, t-1}\right]\end{array}$ & 790 & 805 \\
\hline $\begin{array}{l}\text { Is our model better than one where expectations } \\
\text { are randomly determined? }\end{array}$ & Model with random expectations & 828 & 846 \\
\hline
\end{tabular}


for whatever reason, might be more or less prone to being affected by this impact than suppliers. We included a role dummy, coded 1 if the respondent was a supplier and 0 if the respondent was a buyer. The interaction of this dummy with the quadratic effect of discrepancy had no significant impact on performance outcomes, suggesting that discrepancy operates similarly across the dyad.

Finally, we consider the possibility that the discrepancy quadratic term could be highly correlated with related measures such as trust. Although we have no trust measure at the organizational level, we find that the correlation between the quadratic term and interpersonal trust is moderate $(\rho=-0.34)$, albeit significant. This relatively moderate correlation indicates that the quadratic form of discrepancy is more than just trust, but it also adds some convergent validity to our measure because it is related to other constructs to which it ought to be related. We also estimated a model in which interpersonal trust is treated as a covariate, and there was no substantive change in results.

\section{Discussion}

The results of this research accord with past work suggesting that opportunism has an undermining effect on interorganizational exchange, whereas goal congruence and bilateral idiosyncratic investments have the opposite effects. We extend our understanding of this area in several important ways. First, we demonstrate the negative impact of discrepant behavior, and we show that it holds deleterious consequences for exchange.

Second, the effect of uncertainty is practically the same as the linear deviation effect, implying that the total effect on performance will typically be negative, even if the discrepancy is positive. ${ }^{14}$ Thus, although positive discrepancy is desirable, its unexpectedness may result in significant uncertainty and a negative net effect. Sensemaking would suggest that perhaps the focal firm begins to wonder what precipitated the change in its partner's behavior and, in the worst possibility, becomes suspicious and attributes a dark side to the partner's observed behavior. This negative quadratic effect appears to be manifested similarly across the dyad, for both buyers and suppliers.

Together, our results suggest a response function as illustrated in Figure 1. This figure suggests that any small difference between beliefs and experience, regardless of the direction, would result in a lowered

\footnotetext{
${ }^{14}$ We note that $56 \%$ of the deviation from expectations in our sample of observations was positive in nature, and the remaining $44 \%$ was not.
}

Figure 1 Functional Form of the Net Effect of Discrepancy on Performance

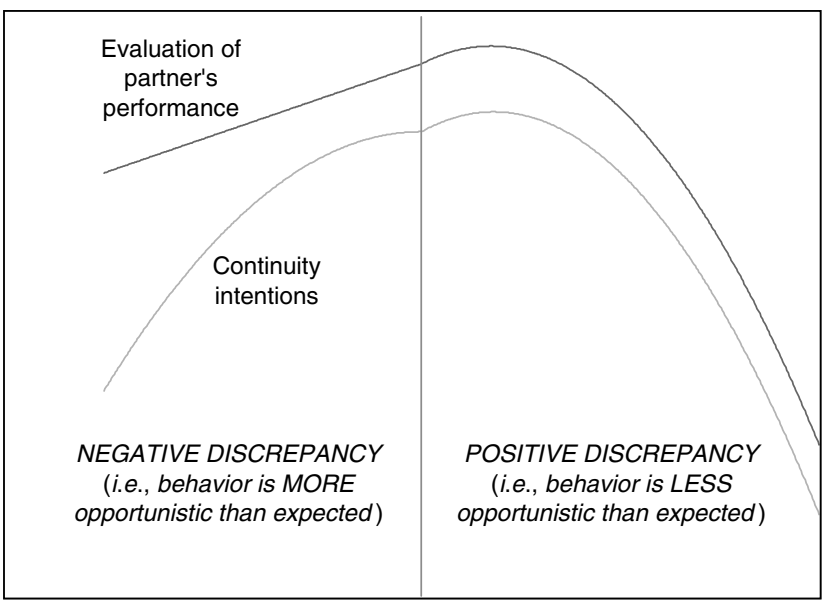

Note. Graph uses data from within the range in our data set.

evaluation. Thus, a belief-based expectation is a stable anchor from which any deviation can negatively impact preference. Why would beliefs show such inflexibility to discrepancy in either direction? Oliver (1976) has argued that this is most likely to occur when the expectation object is particularly important to the individual, as can be the case in interorganizational exchange.

Third, the powerful role of organizational beliefs on exchange performance implies that firms may rely on beliefs even in the presence of current, observed data. This is consistent with the interpretation phase of sensemaking, in which the firm develops or applies ways of comprehending the meaning of the observed information (Daft and Weick 1984). This interpretation is the basis for subsequent action. Thus organizations, like individuals, possess beliefs about covariation between past events and bring those judgments to bear upon a given set of data. Another implication of the finding that belief structures are constraining is that managers (and organizations) may not have an accurate view of reality. This resonates with Vosgerau et al. (2008), who find that, even in long-standing relationships, companies do not know how accurate their perceptions are, even when they believe that they are correct. It may be that belief structures constrain perceptions in a way that primarily supports a negative view of the exchange relationship. This may explain why relationships that hit a downward spiral can continue to cycle out of control and why it is difficult to break out of these dysfunctional patterns. A final implication is that belief discrepancy, in small doses, has less of an undermining effect on the relationship. Perhaps the way to break a spiral of suspicion is to engage in smaller, incremental changes in behavior, and over time, these actions might result in less adverse consequences. 
Fourth, we identify the boundary conditions of the negative effects of belief discrepancy. Firms that are more dependent will tolerate discrepancy (i.e., the functional form in the positive domain shifts right in Figure 1). As the exchange matures, the impact of incoherence is strengthened (i.e., the functional form shifts left in the figure). Perhaps as relationships mature, there is less flexibility or change in belief structures. A history of working together creates a shared identity in which the parties learn that cooperation and mutual undertaking can benefit the exchange as a whole. When incoherence occurs, the effect stands in stark contrast to beliefs that have been widely held, and this is corrosive. Furthermore, bilateral investments are found to play a critical role in safeguarding relationships against behavioral incoherence. This echoes the findings of Jap and Anderson (2003), who find that even as ex post opportunism in exchange increases, the mutual "tying of the hands" can be a powerful mitigation of relationship poison.

Together, these results underscore the value of market experience; specifically, general market experience creates a strong frame of reference that filters all new experiences and observations and impacts relationship performance. In contrast to studies that show that a greater accumulation of exchange history leads to greater expectations of continuity (Poppo et al. 2008), we find that this effect can be reversed in the presence of opportunism discrepancy and incoherence. Surprisingly, a history of exchange is not always a positive buffer against relationship deterioration; in fact, such relationships are actually more prone to the deleterious effects of uncertainty than less developed relationships.

\section{Managerial Implications}

Collectively, this research extends our understanding of how relationships are undermined through organizational belief structures, processes, and behaviors. We generate implications for protecting key exchanges from the dark side of long-term relations. First, we suggest that quick, particularly drastic changes in behavior are unhealthy for the relationship and may in fact be met with suspicion. This echoes the findings of Jap and Anderson (2007), who find that relationships have difficulty recovering from negative histories. Perhaps "good" behavior inconsistent with expectations is less understandable than "bad" behavior consistent with expectations. Relationship managers might be better off addressing the uncertainty generated, even by positive actions. It might be better to confront the partner with concerns rather than allow the uncertainty to languish. This is a key trade-off in relationship management, as firms attempt to balance both the sunny side of positive behavioral deviation from expectations and the dark-side effects of uncertainty. Improving relationships or recovering from incoherence will likely be a slow process that requires steady efforts over time.

The results underscore the value of bilateral, investments in mitigating the negative impact of behavioral incoherence. Mutual economic stakes provide a strong motivation to act in ways supportive of the joint exchange (Anderson and Weitz 1992). Last, managers should be aware that held belief structures may be "an enemy of organizations" (Walsh and Ungson 1991, p. 71), inhibiting learning processes, hiding each party's true intentions, and compromising relationship performance. One path for circumvention might well be to keep short memories.

\section{Future Research}

Whereas opportunism incoherence creates uncertainty and undermines performance, the opposite might be true with more supportive norms such as trust and cooperation; perhaps the overall impact of trust remains positive, albeit reduced. These possibilities and more remain to be explored. Furthermore, the dynamic process of updating belief structures is worthy of further investigation. More longitudinal observations and innovative methods are required to enable a richer understanding of the phenomena. Another avenue for future work would be the alternative specifications for how incoherence manifests, particularly at the interorganizational and interpersonal levels. Campbell's (1958) notion of entitativity, or the degree of correspondence between individual exemplars and a group identity, represents a promising direction.

In sum, we have identified a novel mechanism of relationship dissolution. Our results indicate that discrepancy and incoherence can undermine present and future states of the exchange. Fortunately, its effects can be mitigated via the organizational structure of the interorganizational relationship (i.e., power asymmetry, bilateral idiosyncratic investments, and relationship stage). By observing the consequences of discrepancy, incoherence, and the conditions under which these effects are inhibited, we are able to infer something useful about the seeds of interorganizational dissolution. We might also have stimulated thinking on how to better structure exchange and manage it on an ongoing basis.

\section{Acknowledgments}

This project was supported by grants from the Marketing Science Institute (4-882), the Goizueta Business School, and the Alden G. Clayton Dissertation Competition. Data support was provided by the Institute for the Study of Business Markets, Pennsylvania State University. The authors are grateful to Ulf Bockenholt, Rajdeep Grewal, Gary Lilien, Steve Stern, Michael Martin, the Kellogg Marketing Camp, and the editorial review team for helpful comments on earlier drafts. 


\section{Appendix. Construct Measures}

All measures are 7-point Likert-type scale anchored from strongly disagree $=1$ to strongly agree $=7$, unless otherwise noted. "They" and "us" refer to the two firms, the buyer and supplier together. (R) denotes reversed score.

Overall Evaluation of Partner's Performance (PERFEVAL) $(\alpha=0.88)$, adapted from Kumar et al. (1992)

Our association with this supplier/buyer has been a highly successful one.

The supplier/buyer leaves a lot to be desired from an overall performance standpoint.

If we had to give the supplier a performance appraisal, it would be outstanding.

Overall, the results of our relationship with the supplier/buyer have fallen short of our expectations. (R)

Continuity Intentions (CONTIN) $(\alpha=0.87)$, adapted from Jap and Anderson (2003)

Our relationship with this firm will last far in to the future.

We expect to continue working with this firm on a long-term basis.

We expect to terminate our relationship with this firm soon. (R)

Goal Congruence of the Dyad (GOAL) ( $\alpha=0.68$ in time 1 and $\alpha=0.72$ in time 2), adapted from Jap (1999)

They support each other's objectives. They have different goals. (R)

Observed Opportunism (OPPT) ( $\alpha=0.90$ in time 1 and $\alpha=0.91$ in time 2), adapted from Jap and Anderson (2003)

When a problem occurs, how often will the buyer (supplier) do the following? $(1=$ hardly ever, $7=$ very often)

They make hollow promises. They are unwilling to accept responsibility.

They are aloof toward us. They make false accusations.

They "window dress" their efforts to improve. They provide false information.

They expect us to pay for more than our fair share They fail to provide proper notification.

of the costs to correct the problem.

Own Dependence on Other Firm (DEPEND) $(\alpha=0.70)$, adapted from Jap and Ganesan (2000)

This supplier/buyer is critical to our future performance.

Our firm is dependent on this supplier/buyer.

This supplier/buyer is important to our financial performance.

Relationship Phase (RELPHASE) from Jap and Ganesan (2000)

Relationships typically evolve through a number of phases over time. Which of the following best describes your firm's current relationship with $\mathrm{X}$ ? (Check only one)

Exploration-Both firms are discovering and testing the goal compatibility, integrity, and performance of the other as well as potential obligations, benefits, and burdens involved with working together on a long-term basis.

Buildup-Both firms are receiving increasing benefits from the relationship and a level of trust and satisfaction has been developed such that they are more willing to become committed to the relationship on a long-term basis.

Maturity-Both firms have an ongoing, long-term relationship in which both are receiving acceptable levels of satisfaction and benefits from the relationship.

Decline-One or both members have begun to experience dissatisfaction and is contemplating relationship termination, considering alternative manufacturers or customers, and is beginning to communicate an intent to end the relationship.

Deterioration-The firms have begun to negotiate terms for ending the relationship and/or are currently in the process of dissolving the relationship.

Bilateral Idiosyncratic Investments (INVEST) $(\alpha=0.82)$, adapted from Anderson and Weitz (1992)

If this relationship were to end, they would be wasting a lot of knowledge that's tailored to their relationship.

If either company were to switch to a competitive buyer or vendor, they would lose a lot of the investments made in the present relationship.

They have invested a great deal in building up their joint business.

They have developed procedures, routines, and understanding tailored to their relationship.

Problem Frequency (PROBFREQ)

How frequently do problems arise between your firm and this firm? ( $1=$ hardly ever, $7=$ very often)

Interpersonal Mutual Trust (TRUST) $(\alpha=0.92)$, adapted from Jap (1999)

We are very honest in dealing with each other.

We trust each other.

We consider each other's interests when problems arise. 


\section{References}

Anderson, E., B. Weitz. 1989. Determinants of continuity in conventional industrial channel dyads. Marketing Sci. 8(4) 310-323.

Anderson, E., B. Weitz. 1992. The use of pledges to build and sustain commitment in distribution channels. J. Marketing Res. 29(1) 18-34.

Armstrong, J. S., T. S., Overton. 1977. Estimating nonresponse bias in mail surveys. J. Marketing Res. 14(3) 396-402.

Arrow, K. J. 1971. Essays in the Theory of Risk-Bearing. Elsevier, New York.

Artz, K. W., T. H. Brush. 2000. Asset specificity, uncertainty and relational norms: An examination of coordination costs in collaborative strategic alliances. J. Econom. Behav. Organ. 41(4) 337-362.

Baker, W. E., R. R. Faulkner, G. A. Fisher. 1998. Hazards of the market: The continuity and dissolution of interorganizational market relationships. Amer. Sociol. Rev. 63(2) 147-177.

Bercovitz, J., S. D. Jap, J. A. Nickerson. 2006. The antecedents and performance implications of cooperative exchange norms. Organ. Sci. 17(6) 724-740.

Broniarczyk, S. M., J. W. Alba. 1994. The role of consumers' intuitions in inference making. J. Consumer Res. 21(3) 393-407.

Brown, J. R., C. S. Dev, D.-J. Lee. 2000. Managing marketing channel opportunism: The efficacy of alternative governance mechanisms. J. Marketing 64(2) 51-65.

Campbell, D. T. 1958. Common fate, similarity, and other indices of the status of aggregates of persons as social entities. Behav. Sci. 3(1) $14-25$.

Chiles, T. H., J. F. McMackin. 1996. Integrating variable risk preferences, trust, and transaction cost economics. Acad. Management Rev. 21(1) 73-99.

Crampton, S. M., J. A. Wagner. 1994. Percept-percept inflation in microorganizational research: An investigation of prevalence and effect. J. Appl. Psych. 79(1) 67-76.

Daft, R. L., K. E. Weick. 1984. Toward a model of organizations as interpretation systems. Acad. Management Rev. 9(2) 284-295.

Dick, A., D. Chakravarti, G. Biehal. 1990. Memory-based inferences during consumer choice. J. Consumer Res. 17(1) 82-93.

Duck, S. W. 1981. Towards a research map for the study of relationship breakdown. S. Duck, R. Gilmour, eds. Personal Relationships: Personal Relationships in Disorder, Vol. 3. Academic Press, London, 1-29.

Dwyer, F. R., P. H. Schurr, S. Oh. 1987. Developing buyer-seller relationships. J. Marketing 51(2) 11-27.

Einhorn, H. J., R. M. Hogarth. 1985. Ambiguity and uncertainty in probabilistic inference. Psych. Rev. 92(4) 433-461.

Eisenhardt, K. M. 1989. Agency theory: An assessment and review. Acad. Management Rev. 14(1) 57-74.

Eliashberg, J., D. A. Michie. 1984. Multiple business goals sets as determinants of marketing channel conflict: An empirical study. J. Marketing Res. 21(1) 75-88.

Fornell, C., D. F. Larcker. 1981. Evaluating structural equation models with unobservable variables and measurement error. J. Marketing Res. 18(1) 39-50.

Geylani, T., J. J. Inman, F. Ter Hofstede. 2008. Image reinforcement or impairment: The effects of co-branding on attribute uncertainty. Marketing Sci. 27(4) 730-744.

Hardie, B. G. S., E. J. Johnson, P. S. Fader. 1993. Modeling loss aversion and reference dependence effects on brand choice. Marketing Sci. 12(4) 378-394.

Heide, J. B., A. S. Miner. 1992. The shadow of the future: Effects of anticipated interaction and frequency of contact on buyer-seller cooperation. Acad. Management J. 35(2) 265-291.

Jap, S. D. 1999. Pie-expansion efforts: Collaboration processes in buyer-supplier relationships. J. Marketing Res. 36(4) 461-475.
Jap, S. D., E. Anderson. 2003. Safeguarding interorganizational performance and continuity under ex post opportunism. Management Sci. 49(12) 1684-1701.

Jap, S. D., E. Anderson. 2007. Testing a life-cycle theory of cooperative interorganizational relationships: Movement across stages and performance. Management Sci. 53(2) 260-275.

Jap, S. D., S. Ganesan. 2000. Control mechanisms and the relationship life cycle: Implications for safeguarding specific investments and developing commitment. J. Marketing Res. 37(2) 227-245.

Jöreskog, K. G., D. Sörbom. 2006. LISREL 8.80. Scientific Software International, Lincolnwood, IL.

Joskow, P. L. 1987. Contract duration and relationship-specific investments: Empirical evidence from coal markets. Amer. Econom. Rev. 77(1) 168-185.

Kayande, U., J. H. Roberts, G. L. Lilien, D. K. H. Fong. 2007. Mapping the bounds of incoherence: How far can you go and how does it affect your brand? Marketing Sci. 26(4) 504-513.

Keeney, R. L., H. Raiffa. 1993. Decisions with Multiple Objectives: Preferences and Value Tradeoffs. Cambridge University Press, Cambridge, UK.

Kopalle, P. K., D. R. Lehmann. 2001. Strategic management of expectations: The role of disconfirmation sensitivity and perfectionism. J. Marketing Res. 38(3) 386-394.

Kumar, N., L. W. Stern, R. S. Achrol. 1992. Assessing reseller performance from the perspective of the supplier. J. Marketing Res. 29(2) 238-253

Lindell, M. K., D. J. Whitney. 2001. Accounting for common method variance in cross-sectional research designs. J. Appl. Psych. 86(1) 114-121.

Lusch, R. F., J. R. Brown. 1996. Interdependency, contracting, and relational behavior in marketing channels. J. Marketing 60(4) 19-38.

Meyers-Levy, J., A. M. Tybout. 1989. Schema congruity as a basis for product evaluation. J. Consumer Res. 16(1) 39-54

Mittal, V., W. T. Ross Jr., P. M. Baldasare. 1998. The asymmetric impact of negative and positive attribute-level performance on overall satisfaction and repurchase intentions. J. Marketing 62(1) 33-47.

Murphy, G. L., D. L. Medin. 1985. The role of theories in conceptual coherence. Psych. Rev. 92(3) 289-316.

Nilakant, V., H. Rao. 1994. Agency theory and uncertainty in organizations: An evaluation. Organ. Stud. 15(5) 649-672.

Oliver, R. L. 1976. Hedonic reactions to the disconfirmation of product performance expectations: Some moderating conditions. J. Appl. Psych. 61(2) 246-250.

Parasuraman, A., V. A. Zeithaml, L. L. Berry. 1985. A conceptual model of service quality and its implications for future research. J. Marketing 49(4) 41-50.

Podsakoff, P. M., S. B. MacKenzie, J. Y. Lee, N. P. Podsakoff. 2003. Common method biases in behavioral research: A critical review of the literature and recommended remedies. J. Appl. Psych. 88(5) 879-903.

Poppo, L., K. Z. Zhou, S. M. Ryu. 2008. Alternative origins to interorganizational trust: An interdependence perspective on the shadow of the past and the shadow of the future. Organ. Sci. 19(1) 39-55.

Rehder, B., R. Hastie. 2004. Category coherence and category-based property induction. Cognition 91(2) 113-153.

Rindfleisch, A., A. J. Malter, S. Ganesan, C. Moorman. 2008. Crosssectional versus longitudinal survey research: Concepts, findings, and guidelines. J. Marketing Res. 45(3) 261-279. 
Searle, S. R., G. Casella, C. E. McCulloch. 1992. Variance Components. John Wiley \& Sons, New York.

Thomas, J. B., S. M. Clark, D. A. Gioia. 1993. Strategic sensemaking and organizational performance: Linkages among scanning, interpretation, action, and outcomes. Acad. Management J. 36(2) 239-270.

Vosgerau, J., E. Anderson, W. T. Ross Jr. 2008. Can inaccurate perceptions in business-to-business (B2B) relationships be beneficial? Marketing Sci. 27(2) 205-224.
Walsh, J. P., G. R. Ungson. 1991. Organizational memory. Acad. Management Rev. 16(1) 57-91.

Wathne, K. H., J. B. Heide. 2000. Opportunism in interfirm relationships: Forms, outcomes, and solutions. J. Marketing 64(4) 36-51.

Weick, K. E., K. M. Sutcliffe, D. Obstfeld. 2005. Organizing and the process of sensemaking. Organ. Sci. 16(4) 409-421.

Williamson, O. E. 1993. Opportunism and its critics. Managerial Decision Econom. 14(2) 97-107. 